\title{
L'évolution de l'agriculture neuchâteloise de 1965 à 1980; problèmes de comparaison
}

\section{Introduction}

Dans le cadre d'une étude de base sur l'agriculture, en vue de l'élaboration du Plan directeur du canton de Neuchâtel, nous rencontrons un certain nombre de problèmes lorsque nous désirons analyser l'évolution des exploitations agricoles.

L'étude en cours sera présentée dans la première partie de l'exposé, alors que nous discuterons quelques problèmes spécifiques dans la seconde.

\section{L'étude en cours}

\subsection{Le contexte de l'étude}

La loi fédérale sur l'aménagement du territoire prévoit des études de base pour déterminer dans les grandes lignes le cours que doit suivre l'aménagement du territoire.

Dans le canton de Neuchâtel, un groupe de travail a été formé par le Service cantonal de l'Aménagement du territoire. Il est composé de représentants et de spécialistes de divers domaines. Chacun de ces représentants est chargé d'organiser des groupes de travail internes pour élargir la discussion dans chacun des domaines.

Ainsi le groupe cantonal faîtier se compose de 9 secteurs qui sont l'agriculture, dont l'étude a été confiée à l'Institut de géographie, la nature et le paysage, la gestion de l'eau, la protection de l'environnement (eau - sol - air - bruit), l'énergie, les transports, l'urbanisme, l'économie et les rives des lacs. Signalons à ce propos que l'Institut de géographie a fait une étude sur la perception du lac et des rives par les "utilisateurs» ainsi que par la population neuchâteloise en général.

Le groupe cantonal de travail se réunit fréquemment afin de construire les bases permettant l'élaboration du Plan directeur.

Le Plan directeur englobe la totalité du territoire cantonal et met en évidence les mesures d'intérêt supérieur. Il devra être terminé en 1985.

Il faut noter que mis à part le décret cantonal sur la protection des sites naturels, le décret sur les biotopes

Cet article a fait l'objet d'une communication lors du symposium d'octobre 1983. et la loi sur la viticulture, l'aménagement du territoire dans le canton de Neuchâtel a été le fait des communes qui ont élaboré elles-mêmes leurs plans d'aménagement.

Le Plan directeur tiendra compte des différents plans communaux légalisés, il tracera notamment le cadre à l'intérieur duquel les communes pourront prévoir l'extension future de leur périmètre de construction.

Un projet de loi cantonale sur l'aménagement du territoire est actuellement en procédure de consultation.

\subsection{Les méthodes de l'étude}

\subsubsection{Introduction}

L'Institut de géographie de Neuchâtel est chargé de l'étude du plan sectorial «agriculture».

Avant de présenter l'étude dans ses grandes lignes, nous devons préciser que nous avons formé un groupe de travail qui est composé de représentants des services du Département de l'agriculture du canton (Chef du Service de l'économie agricole), du Directeur de la Chambre neuchâteloise d'agriculture et de viticulture, du directeur du Service cantonal de vulgarisation agricole, et des responsables de l'Aménagement du territoire. Nous avons également prévu une collaboration étroite avec les représentants du secteur «Nature et Paysage», afin d'aplanir entre nous les différends que nous ne manquerons pas d'avoir. Nous présenterons des projets aussi complémentaires que possibles pour avoir plus de "poids» lors des rencontres du groupe de travail cantonal.

Nous avons pu remarquer récemment, lors du dernier colloque de la Commission d'aménagement rural de l'UGI, que le souci de cohérence face aux autorités est constant chez plusieurs géographes. En effet, nous avons constaté que si une étude veut être prise en considération par un service gouvernemental, elle est obligée de refléter une réflexion tenant compte, de façon précise, des diverses tendances économiques actuelles, et non pas de se cantonner à un credo choisi sur simple conviction éco-politique du mandant ou du mandataire.

Eric F. Berthoud, Institut de géographie de l'Université de Neuchâtel, Avenue Clos-Brochet 30, 2000 Neuchâtel 


\subsubsection{Les objectifs de l'étude}

Cette étude devra permettre de déterminer à moyen terme (1995-2000) les besoins en terrain qui permettront à l'agriculture de jouer les rôles qui lui ont été attribués:

1. Assurer la part de l'agriculture du canton à l'alimentation du pays en cas de crise comme en période de paix.

2. Apport économique au canton:

a) conserver une population paysanne forte,

b) maintien de l'importance des secteurs liés à l'agriculture.

3. Assurer les prestations en faveur de la population non agricole et de l'entretien du territoire, tout en s'efforçant d'éviter de porter atteinte à l'environnement.

Ces divers points méritent quelques développements:

\subsubsection{Assurer l'alimentation du pays}

a) Les terres réservées à la culture en cas de crise doivent être préservées, ceci du point de vue légal et du point de vue de la survie des agriculteurs en économie de paix.

Il est à remarquer que, bien que le canton possède une S.A.U. de 32000 ha, seuls 4657 ha (1980) sont "ouverts» (surface de culture), alors que 8200 ha sont prévus par l'économie de crise.

b) Pour l'instant, l'agriculture de notre canton est basée sur l'élevage et la production laitière. Un changement est difficile, voire impossible. D'autre part, il faut pouvoir maintenir la possibilité de travail à l'agriculture.

1.2.1.2 Apport économique de l'agriculture

Nous ne mentionnerons en passant que:

a) les postes de travail, ainsi que leur stabilité, indispensables à tout investissement à long terme.

b) les postes de travail dans les secteurs en amont (mécanique, etc.) et en aval (transformation des produits laitiers, etc.).

\subsubsection{Autres prestations}

Selon le cinquième rapport sur la situation de l'agriculture, des prestations qui sont parfois en conflit avec les principes de rationalisation de la production:

- Entretien du territoire (n'implique pas une approche «écologique»).

- Maintien des régions rurales.

- Sauvegarde du paysage et préservation des structures de l'habitat.

\subsubsection{L'analyse}

1. Nous analyserons l'évolution de l'agriculture du canton de 1965 à 1984, en nous occupant essentiellement des thèmes suivants:

1. Utilisation du sol

2. Structures d'exploitation

3. Population agricole.
Nous avons dans une première étape mis au point une banque de données qui nous permettra de procéder à une partie importante de l'étude:

l'analyse de l'évolution des exploitations agricoles du canton de 1965 à 1984 .

2. Nous dresserons ensuite le tableau du rôle et de l'importance de l'agriculture en 1984, en insistant sur les thèmes suivants:

1. Apport économique de l'agriculture et des secteurs annexes.

2. Espace vert maintenu par l'agriculture.

3. L'équipement des zones rurales.

\subsubsection{Les perspectives de l'étude}

Il nous faudra délimiter des zones agricoles, certaines pourront sans problème être déclarées "intangibles», d'autres se trouveront être l'objet d'un conflit d'utilisation.

Il sera très utile alors de disposer de divers renseignements recueillis dans la phase d'analyse qui nous permettront d'établir des priorités.

Nous proposerons les critères de décision suivants:

1. Viabilité des exploitations.

2. Rapport avec les secteurs liés (en amont et an aval).

3. Rapport avec les espaces à protéger.

4. Liens entre agriculture et les autres secteurs tel que: l'équipement, le tourisme, etc.

\section{Les problèmes de comparaison}

\subsection{L'évolution de l'agriculture}

\subsubsection{Introduction}

Notre propos n'est pas ici de faire un portrait de l'agriculture suisse et neuchâteloise.

Nous renvoyons les intéressés à quelques ouvrages essentiels: (Conseil Fédéral, 1976), (Kipfer, 1982), (Quartier, 1978) et (Chiffelle, 1968).

Pourtant il est essentiel de mentionner quelques points qui résument la tendance.

\subsubsection{La structure des exploitations}

L'évolution de la structure des exploitations est composée d'un double mouvement.

Le premier, qui est essentiel, est un agrandissement de la surface des exploitations et une diminution du nombre des parcelles.

En Suisse, la surface moyenne a varié de 7,58 ha en 1965 à 9,21 ha en $1980(13,31$ ha et 18 ha respectivement pour le canton de Neuchâtel); mais surtout $42 \%$ (Neuchâtel: 60\%) des exploitations comptaient plus de 10 ha en 1980, alors qu'il n'y en avait que $29 \%$ en 1965 (Neuchâtel comptait plus de $56 \%$ d'exploitations $\mathrm{de}+$ de $10 \mathrm{~km}$ en 1965).

Le second mouvement est une diminution faible du nombre des petites exploitations ( $0-1$ ha). Ceci est dû au maintien d'un nombre élevé d'ouvriers-paysans et de micro-exploitations spécialisées. 
Tableau 1 Evolution de la structure des exploitations agricoles en Suisse et dans le canton de Neuchâtel

\begin{tabular}{|c|c|c|c|c|c|c|c|c|c|c|}
\hline & & 1965 & 8 & 1975 & 8 & 1980 & 8 & $\underline{65-75}$ & $\underline{75-80}$ & $65-80$ \\
\hline $\begin{array}{l}\text { Nombre des exploitations } \\
\text { dont avec ... ha de }\end{array}$ & $\begin{array}{l}\mathrm{CH} \\
\mathrm{NE}\end{array}$ & $\begin{array}{r}162 \cdot 500 \\
2 \cdot 515\end{array}$ & & $\begin{array}{r}133 \cdot 100 \\
2 \cdot 091\end{array}$ & & $\begin{array}{r}125 \cdot 300 \\
1 \cdot 944\end{array}$ & & $\begin{array}{l}-18 \\
-17\end{array}$ & $\begin{array}{l}-6 \\
-7\end{array}$ & $\begin{array}{l}-23 \\
-23\end{array}$ \\
\hline $0-1$ & $\begin{array}{l}\mathrm{CH} \\
\mathrm{NE}\end{array}$ & $\begin{array}{r}30 \cdot 500 \\
569\end{array}$ & $\begin{array}{l}18.7 \\
22.6\end{array}$ & $\begin{array}{r}27 \cdot 400 \\
535\end{array}$ & $\begin{array}{l}20.6 \\
25.6\end{array}$ & $\begin{array}{r}27 \cdot 100 \\
473\end{array}$ & $\begin{array}{l}21.6 \\
24.4\end{array}$ & $\begin{array}{r}-10 \\
-6\end{array}$ & $\begin{array}{r}-2 \\
-12\end{array}$ & $\begin{array}{l}-11 \\
-17\end{array}$ \\
\hline $1-5$ & $\begin{array}{l}\mathrm{CH} \\
\mathrm{NE}\end{array}$ & $\begin{array}{r}44 ' 300 \\
284\end{array}$ & $\begin{array}{l}27.3 \\
11.3\end{array}$ & $\begin{array}{r}30 \cdot 100 \\
200\end{array}$ & $\begin{array}{r}22.6 \\
9.6\end{array}$ & $\begin{array}{r}25^{\prime} 600 \\
206\end{array}$ & $\begin{array}{l}20.4 \\
10.6\end{array}$ & $\begin{array}{l}-32 \\
-30\end{array}$ & $\begin{array}{r}-15 \\
+3\end{array}$ & $\begin{array}{l}-42 \\
-27\end{array}$ \\
\hline $5-10$ & $\begin{array}{l}\mathrm{CH} \\
\mathrm{NE}\end{array}$ & $\begin{array}{r}40 \cdot 000 \\
254\end{array}$ & $\begin{array}{l}24.6 \\
10.1\end{array}$ & $\begin{array}{r}24 \cdot 600 \\
122\end{array}$ & $\begin{array}{r}18.5 \\
5.8\end{array}$ & $\begin{array}{r}20 \cdot 150 \\
98\end{array}$ & $\begin{array}{r}16.2 \\
5.0\end{array}$ & $\begin{array}{l}-38 \\
-52\end{array}$ & $\begin{array}{l}-18 \\
-20\end{array}$ & $\begin{array}{l}-49 \\
-61\end{array}$ \\
\hline $10-20$ & $\begin{array}{l}\mathrm{CH} \\
\mathrm{NE}\end{array}$ & $\begin{array}{r}37 \cdot 000 \\
759\end{array}$ & $\begin{array}{l}22.8 \\
30.2\end{array}$ & $\begin{array}{r}36 \cdot 100 \\
436\end{array}$ & $\begin{array}{l}27.1 \\
20.8\end{array}$ & $\begin{array}{r}34 \cdot 600 \\
317\end{array}$ & $\begin{array}{l}27.6 \\
16.3\end{array}$ & $\begin{array}{r}-2 \\
-43\end{array}$ & $\begin{array}{r}-4 \\
-27\end{array}$ & $\begin{array}{r}-6 \\
-58\end{array}$ \\
\hline $20-50$ & $\begin{array}{l}\mathrm{CH} \\
\mathrm{NE}\end{array}$ & $\begin{array}{r}10 \cdot 000 \\
591\end{array}$ & $\begin{array}{r}6.2 \\
23.5\end{array}$ & $\begin{array}{r}14 \cdot 100 \\
722\end{array}$ & $\begin{array}{l}10.6 \\
34.5\end{array}$ & $\begin{array}{r}16 \cdot 800 \\
735\end{array}$ & $\begin{array}{l}13.4 \\
37.8\end{array}$ & $\begin{array}{l}+41 \\
+22\end{array}$ & $\begin{array}{r}+19 \\
+2\end{array}$ & $\begin{array}{l}+68 \\
+24\end{array}$ \\
\hline 50 et plus & $\begin{array}{l}\mathrm{CH} \\
\mathrm{NE}\end{array}$ & $\begin{array}{r}700 \\
58\end{array}$ & $\begin{array}{l}0.4 \\
2.3\end{array}$ & $\begin{array}{r}800 \\
76\end{array}$ & $\begin{array}{l}0.6 \\
3.7\end{array}$ & $\begin{array}{r}1 ' 050 \\
115\end{array}$ & $\begin{array}{l}0.8 \\
5.9\end{array}$ & $\begin{array}{r}+4 \\
+31\end{array}$ & $\begin{array}{l}+28 \\
+51\end{array}$ & $\begin{array}{l}+47 \\
+98\end{array}$ \\
\hline Moyenne par exploitation & $\begin{array}{l}\mathrm{CH} \\
\mathrm{NE}\end{array}$ & $\begin{array}{r}7.58 \\
13.31\end{array}$ & & $\begin{array}{r}8.98 \\
16.58\end{array}$ & & $\begin{array}{r}9.21 \\
17.89\end{array}$ & & $\begin{array}{l}+18 \\
+25\end{array}$ & $\begin{array}{l}+6 \\
+8\end{array}$ & $\begin{array}{l}+22 \\
+34\end{array}$ \\
\hline Surface agricole utile & $\begin{array}{l}\mathrm{CH} \\
\mathrm{NE}\end{array}$ & $\begin{array}{r}1 ' 231 ' 505 \\
33^{\prime} 474\end{array}$ & & $\begin{array}{r}1 \cdot 195 ' 476 \\
32 \cdot 731\end{array}$ & & $\begin{array}{r}1 ' 145 ' 493 \\
34 ' 177\end{array}$ & & $\begin{array}{l}-3 \\
-2\end{array}$ & $\begin{array}{l}-4 \\
+4\end{array}$ & $\begin{array}{l}-7 \\
+2\end{array}$ \\
\hline Terres ouvertes & $\begin{array}{l}\mathrm{CH} \\
\mathrm{NE}\end{array}$ & $\begin{array}{r}248 \cdot 901 \\
4 ' 536\end{array}$ & $\begin{array}{l}20.2 \\
14\end{array}$ & $\begin{array}{r}262 \cdot 530 \\
4 \cdot 447\end{array}$ & $\begin{array}{l}22 \\
14\end{array}$ & $\begin{array}{c}274 \cdot 780 \\
4 \cdot 657\end{array}$ & $\begin{array}{l}24.1 \\
14\end{array}$ & $\begin{array}{l}+5 \\
-2\end{array}$ & $\begin{array}{l}+5 \\
+5\end{array}$ & $\begin{array}{r}+10 \\
+3\end{array}$ \\
\hline $\begin{array}{l}\text { Exploitations dirigées } \\
\text { à titre principal }\end{array}$ & $\begin{array}{l}\mathrm{CH} \\
\mathrm{NE}\end{array}$ & $\begin{array}{r}100 \cdot 500 \\
1 \cdot 788\end{array}$ & $\begin{array}{l}62 \\
71\end{array}$ & $\begin{array}{rl}77 & \cdot 800 \\
1 & ' 371\end{array}$ & $\begin{array}{l}58 \\
66\end{array}$ & $\begin{array}{r}72 \cdot 300 \\
1 \cdot 284\end{array}$ & $\begin{array}{l}58 \\
66\end{array}$ & $\begin{array}{l}-23 \\
-23\end{array}$ & $\begin{array}{l}-6 \\
-6\end{array}$ & $\begin{array}{l}-28 \\
-28\end{array}$ \\
\hline
\end{tabular}




\subsection{Les problèmes de comparaison}

\subsubsection{Comparaisons entre exploitations}

Pour l'établissement de notre banque de donnée comparative 1965-1980, les données dont nous pouvons disposer sont de deux types:

1. Fichier sur cartes perforées établies par F. Chiffelle sur la base du recensement des exploitations agricoles de 1965.

2. Les fichiers sur bande magnétique, mise à notre disposition par l'Office statistique fédéral, pour les recensements de 1975 et 1980.

Pour identifier une exploitation, le premier fichier nous indique: la commune, le numéro d'exploitation et le nom de l'exploitant, les deux autres fichiers comportent la commune et un numéro d'exploitation.

Malheureusement, les numéros d'exploitation ont changé au cours des années, puisqu'ils sont établis par les communes ou les agents recenseurs, selon des critères arbitraires. Toute comparaison directe entre les fichiers est donc impossible.

Le seul moyen de remédier à ce problème est le suivant: Procéder à la lecture des copies de recensements 1975 et 1980 et confronter les exploitations une à une avec les données de 1965 .

Heureusement nous possédons soit sur microfilm (1975) soit sur photocopies (1980) - (autorisées) -, les copies de ces feuilles de recensements.

C'est pour prévenir dorénavant ce genre de tracas que l'Office fédéral de la statistique met au point un système de comparaison qui entrera en vigueur lors du recensement fédéral des entreprises de 1985.

Ce système est en cours de contrôle auprès des divers cantons, il permettra de déconnaître la commune, l'exploitant, l'exploitation et l'établissement (une distinction sera donc possible entre les diverses «exploitations» (établissements) d'un même exploitant). Evidemment que seul un fichier central comportera les indications personnelles (nom, prénom, adresse, etc.) et que le numéro codé sera seul distribué, assurant ainsi la protection de la sphère privée.

Dans l'attente de l'établissement de ce système, nous sommes contraints d'accomplir un travail que certains qualifieraient de bénédictin, car il impose la comparaison de données de plus de 6000 exploitations.

\subsubsection{Comparaisons entre variables}

$\mathrm{Au}$ cours des recensements, un certain nombre de variables ont été modifiées.

Dans les grandes lignes, nous indiquerons une irrégularité en ce qui concerne les renseignements demandés au sujet de:

1. La composition des ménages des agriculteurs

2. La collaboration entre agriculteurs

3. Les modes d'acquisition et les formes de propriété

4. Les contrats de production/vente.

Plus en détail, un certain nombre de variables reflètent une évolution de l'agriculture, surtout en ce qui concerne les domaines suivants:
1. Utilisation du sol: certaines variables disparaissent: seigle de printemps, carottes, pois à battre, roses, arbustes et champignons.

D'autres se modifient: les variables poissons et tourbières deviennent étang et marais.

Certaines variables deviennent plus précises, le froment de printemps se divise entre semis de printemps et semis d'automne et on distingue aussi entre prairies artificielles annuelles et bisannuelles.

2. Cheptel: les précisions sont abandonnées concernant les ovins et les caprins.

3. Machines: à part l'apparition de nouvelles machines, on assiste à l'abandon de spécification des systèmes d'étable et des types de carburant.

4. Zones de production: les zones de production animale et végétale ont non seulement varié, mais on assiste à un sérieux changement de nomenclature. Alors qu'en 1965, le seul critère était celui d'appartenance à la zone de montagne, le système proposé pour 1985 (illustré par le tableau no 2), prévoit 11 zones dans lesquelles les zones de productions se chevauchent.

\subsection{L'évolution de la politique agricole au niveau fédéral}

\subsubsection{Introduction}

Au cours de ces dernières années, le rôle de l'agriculture s'est modifié.

Le cinquième rapport sur l'agriculture (CONSEIL FÉDÉRAL, 1976) nous informe du "système d'objectifs» de la politique agricole suisse. Ce système se base sur l'article constitutionnel $31^{\text {bis }}$ et les principes de la loi sur l'agriculture qui stipulent:

"conserver une population paysanne forte et faciliter l'approvisionnement du pays en assurant la production agricole.»

Ce rapport considère comme objectif essentiel, l'exécution des tâches de l'agriculture au service de l'économie publique. Les trois tâches principales de l'agriculteur sont les suivantes:

a) l'approvisionnement des denrées alimentaires en temps normal

b) les préparatifs en vue d'assurer le ravitaillement en période de crise et maintien à long terme du potentiel de production (voir ci-dessous 2.4)

c) l'entretien du territoire (préservation des structures d'habitat aussi bien qu'entretien proprement dit).

En matière d'approvisionnement, la politique suisse vise à l'autonomie complète du pays en cas de crise. Les plans se succèdent qui tentent de prévoir des situations hypothétiques. Ces plans nous intéressent dans la mesure où ils font souvent figure de loi et sont une garantie très importante du maintien d'une surface de production agricole. 
Tableau 2 Numérotation des zones

\begin{tabular}{|c|c|c|c|c|}
\hline Appelation des zones & Abréviation & \multicolumn{3}{|c|}{ Système de codification } \\
\hline & & $1: 2$ & 3 & 4 \\
\hline (1 imite standard) & LS & & & \\
\hline Plaine & $P$ & $\begin{array}{lll} & 1 & 1\end{array}$ & & \\
\hline 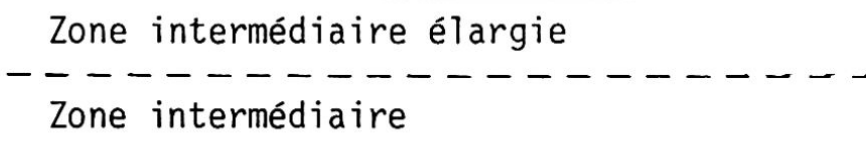 & $\begin{array}{l}\mathrm{ZIE} \\
\mathrm{ZI}\end{array}$ & $\begin{array}{lll}+2 & 1 \\
-2 & 2 & 2\end{array}$ & 眹㛺 & \\
\hline Régions d'élevage contiguës & REC & & & \\
\hline Zone préalpine des collines & ZPC & 41 & & \\
\hline $\begin{array}{l}\text { Zone de montagne } 1 \text { (Cadastre de la pro- } \\
\text { Zone de montagne } 2 \\
-\frac{\text { duction animale) }}{\text { Zone de montagne } 3} \\
\text { Zone de montagne } 4\end{array}$ & $\begin{array}{l}\text { CPA } 1 \\
\overline{C P A} \overline{2} \\
\bar{C}-\overline{-} \\
\overline{C P A} \overline{3}\end{array}$ & 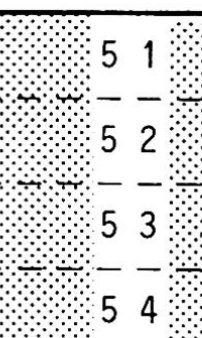 & & 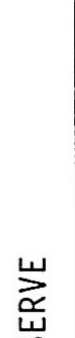 \\
\hline Zone de montagne (1imite standard) & M LS & & & 岗 \\
\hline
\end{tabular}

La numérotation des zones est basée sur un système de code à quatre positions

Chaque position est reliée aux différents groupes de zones de la façon suivante:

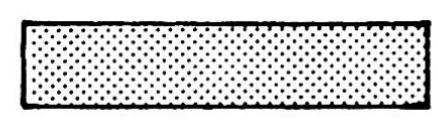

Position 1 et 2 (pour les zones de base)

- Plaine

- Zones créées pour développer la culture des champs

- Zones du cadastre de la production animale (sans les régions d'élevage contiguës)

(ZPC;CPA 1, $2,3$ et 4$)$

Position 3 (pour les zones chevauchantes)

- Régions d'élevage contiguës

(REC)

- Cadastre de la production

(limite standard)

Position 4 (Réserve)

Source: Note de 1'Office fédéral de 1'agriculture du 27.12.1982 
Le plan d'alimentation (VON AH, 1982) prévoit qu'une surface de 450000 ha doit être cultivable. La surface actuelle (1981) des terres ouvertes ne s'élève qu'à 278090 ha. Il est donc urgent d'augmenter cette surface. Le Programme de production agricole (CRIA, 1981) prévoit d'atteindre les 300000 ha en 1990.

$\mathrm{Si}$ la production agricole suisse assure dans certains domaines l'autonomie du pays et permet l'exportation de certaines denrées (produits laitiers p.ex.), elle le doit à l'importation de denrées fourragères. La production de ces denrées importées recouvre actuellement une surface globale de $3000 \mathrm{~km}^{2}$, donc plus que la surface des terres actuellement ouvertes en Suisse.

\subsection{L'apport de la Loi fédérale sur l'aménagement du territoire aux milieux ruraux et agricoles}

\subsubsection{Introduction}

Nous partageons avec Ch. Darbell'zy (DARBELlaY, 1982), l'idée que bien des espoirs sont permis aux agriculteurs par la Loi fédérale sur l'aménagement du territoire. Il faudra aussi que les milieux ruraux acceptent de jouer les rôles que leur donne cette loi.

Nous allons brièvement voir en quoi consistent les attentes et les tâches des agriculteurs face à la LAT.

\subsubsection{Les attentes des agriculteurs}

- Réduction de l'emprise urbaine. La perte quotidienne de 10 ha de surface agricole (l'équivalent d'une exploitation agricole moyenne) devrait être fortement freinée.

En effet, l'article 3 (alinéa 2a) indique: «Il convient notamment de réserver à l'agriculture suffisamment de bonnes terres cultivables.»

- Soustraction des terres cultivables à la concurrence des prix bien plus élevés dont bénéficient les terrains à bâtir, ce qui rend l'accession à la propriété quasi impossible aux agriculteurs. Cette perspective est permise en particulier par l'obligation de désigner des «parties du territoire» qui se prêtent à l'agriculture, où seules seront tolérées les constructions et installations directement nécessaires à l'exploitation agricole du sol (Art 6, 2a).

- Doter les terres agricoles d'une garantie d'affectation. Cette garantie valorisera les efforts consentis à la fois par les exploitants et les pouvoirs publics en vue de l'amélioration durable des exploitations. La loi prévoit des "plans d'affectation» qui délimitent des «zones agricoles». Ces dernières comprennent

a) «les terrains qui se prêtent à l'exploitation agricole ou horticole.»

Ce sont les meilleures terres agricoles.

b) «les terrains qui, dans l'intérêt général, doivent être utilisés par l'agriculture.»

Ces terrains sont situés dans des zones où l'agriculture elle-même ne suffit pas à faire vivre toute une région, mais où elle joue un rôle d'intérêt public (Art 16).

Cette dernière décision permet aussi d'espérer un soutien effectif au combat inégal que le milieu rural ne cesse de mener pour lutter contre un déclin par la création d'emplois, de services et d'équipements.

\section{Conclusion}

Le thème principal de notre étude est bien celui de l'évolution de l'agriculture et en cela nous devons être à même de pouvoir comparer les états de cette économie à diverses époques.

Mais en fait nous pouvons remarquer que pour dessiner à moyen terme la situation de l'agriculture, il nous faut avant tout dégager un certain nombre de types d'évolutions que nous classerons en trois grandes catégories:

1. l'évolution des techniques mises à la disposition des agriculteurs (équipement, semences, sélection, formation, etc.)

2. l'évolution des structures de production et d'exploitation (marché des produits, marché foncier, etc.)

3. l'évolution de la politique agricole de nos gouvernements ainsi que du "rôle idéologique» attribué à l'agriculture (le paysan est garant du maintien de l'environnement, des structures d'habitat, de services, de traditions, d'un "genre de vie»).

Il nous reste donc à tenter de savoir lequel de ces facteurs (technique, structure, politique) est prépondérant sur les autres.

\section{Bibliographie}

CHIFFELLE, Frédéric (1968): Le Bas-Pays neuchâtelois. Etude de géographie rurale. La Baconnière, Neuchâtel.

CHIFFELLE, Frédéric (1983): Swiss Agricultural Policy in Mountainous Areas. In: Nordia 17/1 pp 27-31.

Conseil Fédéral. Constitution fédérale, Berne.

Conseil Fédéral (1951): Loi fédérale sur l'agriculture, Berne.

Conseil Fédéral (1976): Cinquième rapport sur l'agriculture, Berne.

Conseil Fédéral (1976): Loi fédérale sur l'aménagement du territoire, Berne.

CRIA - Centre romand d'informations en agriculture et alimentation (1981): Programme de production agricole, Lausanne.

DARBELLAY, Charly (1982): “L'agriculture et l'aménagement du territoire." (Cours de I'Association suisse des Ingénieurs agronomes et des ingénieurs en technologie alimentaire), Gümligen. 
DFJP/OFAT - Département fédéral de justice et police - Office fédéral de l'aménagement du territoire (1981): Etude relative à la Loi fédérale sur l'aménagement du territoire, Berne.

DFJPIOFAT - Département fédéral de justice et police - Office fédéral de l'aménagement du territoire (1982): La bataille pour le sol, Berne.

Grand Conseil de la République et Canton de Neuchâtel (1982): Projet de Loi cantonale sur l'aménagement du territoire, Neuchâtel.

JEANNERET, André (1981): “Aménagement du territoire. Plan directeur cantonal.» In: Bulletin de la Société neuchâteloise de géographie, Neuchâtel.
KIPFER, Werner (1982): L'agriculture suisse, SVA, Lindau ZH.

Office fédéral de la statistique (1972): Statistique de la superficie de la Suisse, Berne.

Office fédéral de la statistique (1965, 1975, 1980): Statistiques de la Suisse, Berne (Série dans laquelle sont publiés les résultats des recensements fédéraux).

QUARTIER, Claude (1978): Paysarıs d'aujourd'hui en Suisse, Payot, Lausanne.

VON AH, J. (1982): PA 80 - Planification alimentaire en vue d'assurer le ravitaillement en période de crise, OFDP-OFA, Berne. 